\title{
Synthesis of selenium nanorods with assistance of biomolecule
}

\author{
S KANNAN ${ }^{1}$, K MOHANRAJ ${ }^{1, *}$, K PRABHU ${ }^{1}$, S BARATHAN ${ }^{2}$ and G SIVAKUMAR ${ }^{2}$ \\ ${ }^{1}$ Department of Physics, Manonmaniam Sundaranar University, Tirunelveli 627 012, India \\ ${ }^{2}$ Department of Physics, Annamalai University, Annamalai Nagar 608 002, India
}

MS received 2 September 2013; revised 28 December 2013

\begin{abstract}
Nanorods of one-dimensional (1D) trigonal selenium (t-Se) are synthesized using biomolecule substances for five different aging times $(1 \mathrm{~h}, 2 \mathrm{~h}, 3 \mathrm{~h}, 1$ day and 4 days) by precipitation method. XRD analysis indicates a shift of the (101) plane towards higher diffraction angle for 1 day aging time. It is observed that the crystallite size decreases with increase in aging time except for an aging period of 4 days. FTIR analysis confirmed that the presence of stretching and bending vibrations of Se-O in both synthesized and commercial selenium samples at 465 , 668 and $1118 \mathrm{~cm}^{-1}$. The FESEM micrographs are evident for the changes of rod size as a function of aging time. It is observed that the optical band gap energy is increased with aging time up to 1 day, whereas it decreases in 4 days aging time.
\end{abstract}

Keywords. Selenium; nanorods; $L$-arginine; precipitation method.

\section{Introduction}

Selenium (Se) is a highly demanding element for various applications such as glass manufacturing, pigments, agricultural feed additives, chemical uses and pharmaceuticals. It is naturally available element in various isotropic forms $\left({ }^{74} \mathrm{Se},{ }^{76} \mathrm{Se},{ }^{77} \mathrm{Se},{ }^{78} \mathrm{Se},{ }^{80} \mathrm{Se}\right.$ and $\left.{ }^{82} \mathrm{Se}\right)$ and in different allotropic forms such as hexagonal, trigonal, $\alpha$-monoclinic, $\beta$-monoclinic and amorphous (Brown 2000). Recently, onedimensional (1D) Se nanostructures are widely used in biosensor and solar cells (Zhang et al 2004, 2006c) and Sebased semiconducting nanorods are also preferentially used in photovoltaic devices due to their huge interface, nonflat morphology and band transport (Kislyuk and Dimitriev 2008). Many researchers have synthesized 1D Se nanostructure via reduction process using various selenium sources such as $\mathrm{H}_{2} \mathrm{SeO}_{3}, \mathrm{Na}_{2} \mathrm{SeO}_{3}, \mathrm{Na}_{2} \mathrm{SeSO}_{3}$ and bulk Se powder with different reducing agents such as $\mathrm{N}_{2} \mathrm{H}_{4}$ and $\mathrm{NaCH}_{2} \mathrm{O}$ by hydrothermal (Zhang et al 2006a,b,c), solvothermal (Huang and Qi 2009), solid-solution-solid (Liu et al 2008; Zhu et al 2006), electrodeposition (Zhang et al 2006c), refluxing (Shah et al 2007) and sonochemical method (Ma et al 2004).

To avoid the use of toxic reducing agents, some researchers have used biomolecule substances such as oleic acid, $\beta$-cyclodextrin and cytochrome $\mathrm{C}_{3}$ as stabilizers and ascorbic acid as reducing agent for synthesis of Se nanostructures (Abdelouas et al 2000; Li and Yam 2006; Liu et al 2008). Moreover, $\beta$-carotene, polyvinyl alcohol and glucose were also used in dual role as reducers and structural directing agents (Zhang et al 2006a,b,c; Shah et al 2007; Chen et al 2010). Also researchers have used amino acids such as cysteine, glutathione and tyrosine to synthesize Se,

*Author for correspondence (kmohanraj.msu@ gmail.com)
$\mathrm{MoS}_{2}, \mathrm{Bi}_{2} \mathrm{~S}_{3}$ and $\mathrm{PbS}$ nanostructures by hydrothermal and solvothermal methods (Tong et al 2006; Zhang et al 2006a,b; Gao et al 2008; Wang et al 2011; Chen et al 2012). It is to be noted that the amino acids can control the particle size, shape and restrict the formation of agglomeration by the interaction of their functional groups (Choi and Lee 2011). Although, it influences complex product that was formed during synthesis. Therefore, a high temperature is applied to break the van der Waals interaction between the Se spiral chains. Subsequently, large size particles were formed with emission of $\mathrm{H}_{2} \mathrm{~S}$. In addition, the physico-chemical changes in a sealed autoclave are difficult to monitor. Gao et al (2003) have made an attempt to synthesize Se nanoparticles using glutathione by wet-chemical method. However, this method of synthesis has taken more time to precipitate the Se particles. Song et al (2006) have designed a new method for synthesis of Se nanostructures using water and $n$-butyl alcohol interface, and studied the phase transformation of Se. It is observed from the report that the phase transformation was started at an early stage, and however, the following factors such as density of the two liquids, solubility, pressure at interface, gravity, dispersion and interfacial tensile force, etc. taken into account very seriously during synthesis. These factors are major drawbacks of the interface method. According to our literature review, no report found elsewhere on synthesis of $1 \mathrm{D}$ Se nanostructures by simple precipitation method using biomolecular substances: $L$-arginine and ascorbic acid, which are new and potential strategic routes due to their attracting structure and self-assembling role. Hence, in this work, we report the synthesis of 1D Se nanorods using $L$-arginine and ascorbic acid by precipitation method. The biomolecules are more promising materials, natural abundance, very safe to the environment and low cost. 


\section{Experimental}

$0.5 \mathrm{M} \mathrm{SeO}_{2}(98 \%)$ solution $(10 \mathrm{~mL})$ was mixed into $0.31 \mathrm{M}$ $L$-arginine $\left(\mathrm{C}_{6} \mathrm{H}_{14} \mathrm{~N}_{4} \mathrm{O}_{2}, 99 \%\right)$ solution $(10 \mathrm{~mL})$ and stirred vigorously using magnetic stirrer followed by addition of $0.32 \mathrm{M}$ ascorbic acid $\left(\mathrm{C}_{6} \mathrm{H}_{8} \mathrm{O}_{6}, 99 \%\right)$ solution $(10 \mathrm{~mL})$ into the mixture solution $(\mathrm{pH} \mathrm{7)}$. Subsequently, the transparent solution was changed into red colour, which indicated the formation of amorphous Se ( $\alpha$-Se). Then, the $\alpha$-Se was precipitated and filtered using filter paper and then centrifuged. The precipitate was thoroughly washed using ethanol and distilled water (DW) alternately for several times. The resultant product was re-dispersed into ethanol solution for aging in a dark room for the period of $1 \mathrm{~h}, 2 \mathrm{~h}, 3 \mathrm{~h}, 1$ day and 4 days. After aging, the red precipitates are changed into black colour and it was again centrifuged. Finally, the product was thoroughly washed and dried in air at room temperature for overnight. The dried samples were characterized by $\mathrm{X}$-ray diffraction (XRD) patterns using $\mathrm{CuK} \alpha$ radiation in the range of $2 \theta=10-80^{\circ}$ in an X' Pert PRO diffractometer (PANalytical, The Netherlands). FTIR spectra of the samples were recorded using Perkin Elmer, Fourier transform infrared spectrometer (FTIR) in the range of 4000-450 $\mathrm{cm}^{-1}$ with resolution of $4 \mathrm{~cm}^{-1}$. Field emission scanning electron microscope (FESEM, Curl-Zeiss) and UV-Visible spectrophotometer (UV2300-spectrophotometer) were used to record the surface morphology and optical absorption, respectively.

\section{Results and discussion}

Figure 1(a-f) shows the XRD patterns of Se particles for different aging times. The XRD pattern (figure 1a) of asprepared particle shows amorphous $(\alpha-\mathrm{Se})$ in nature, however, a peak shown at $2 \theta=38.46^{\circ}$ indicates the presence of impurity. It is observed from $1 \mathrm{~h}$ pattern (figure $1 \mathrm{~b}$ ) that two peaks emerging at $2 \theta=23.56^{\circ}$ and $29.72^{\circ}$ are assigned to ( $\left.\begin{array}{lll}1 & 0 & 0\end{array}\right)$ and $\left(\begin{array}{lll}1 & 0 & 1\end{array}\right)$ planes, respectively, of trigonal selenium (t-Se) (JCPDS card no. 06-0362). It is a starting phase transformation of $\alpha$-Se, which is more soluble in ethanol medium. It is to be noted that the phase transformation from $\alpha$-Se into $\mathrm{t}$-Se is possible at about $6.63 \mathrm{~kJ} / \mathrm{mol}$, the glass transition temperature is around $31^{\circ} \mathrm{C}$ and thus, slightly forcing reaction condition or temperature will lead to a phase transformation (Zingaro and Copper 1974).

At 2 and $3 \mathrm{~h}$ aging times (figure $1 \mathrm{c}$ and d), the t-Se characteristics peaks (at $2 \theta=23.56$ and $29.72^{\circ}$ ) are increased in intensity and many crystalline peaks are also shown at $41.33^{\circ}$, $43.64^{\circ}, 45 \cdot 40^{\circ}, 48 \cdot 17^{\circ}, 51 \cdot 68^{\circ}, 55 \cdot 57^{\circ}, 56 \cdot 12^{\circ}, 61 \cdot 20^{\circ}, 61.69^{\circ}$, $65.31^{\circ}, 68.33^{\circ}, 71.58^{\circ}$ and $76.99^{\circ}$, which are assigned to

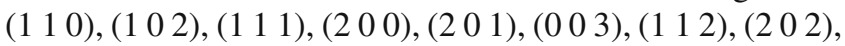
(1 03 ), , (2 10 ), , (2 11 1), (1 113 ) and (2 12 2) planes, respectively, of t-Se. As time proceeds, the intensity of the crystalline peaks increases in 1 day aging (figure $1 \mathrm{e}$ ) than $3 \mathrm{~h}$ aging, while it decreases in 4 days aging (figure 1f) and it is observed that no trace of impurity in the aged samples, which

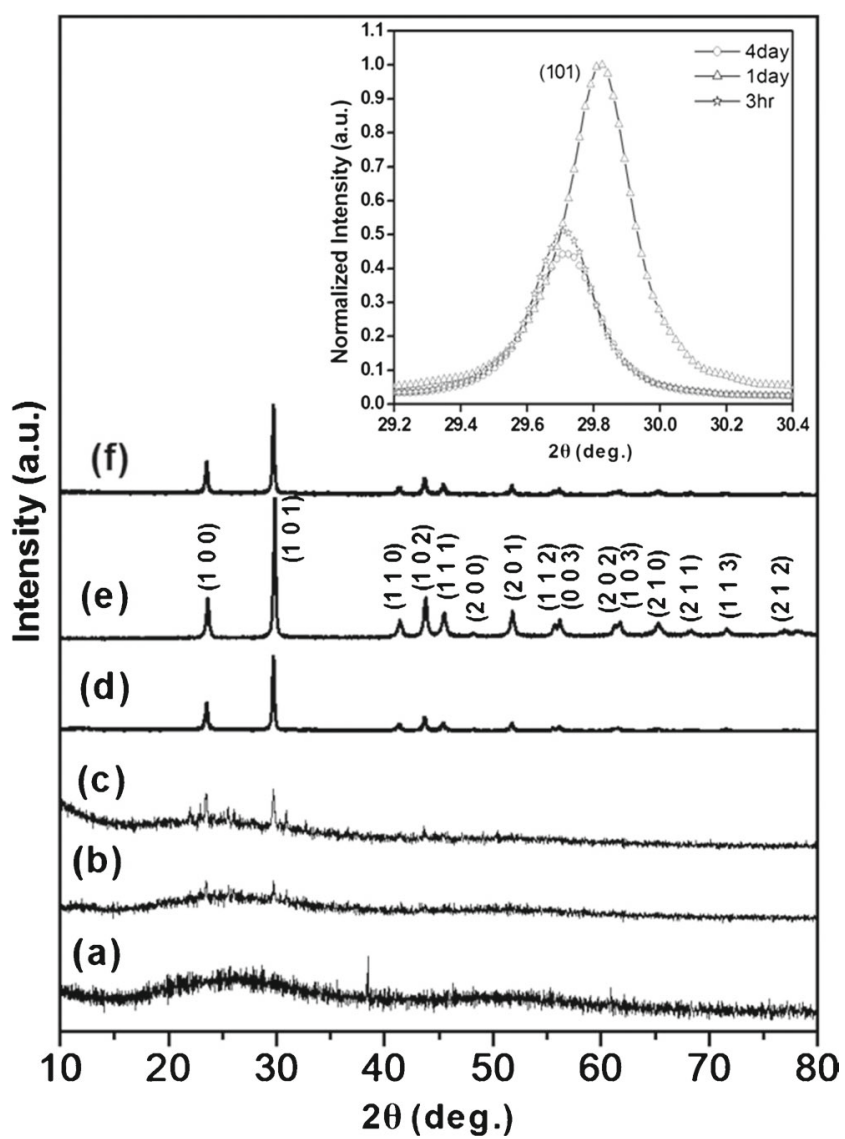

Figure 1. XRD patterns of Se nanoparticles: (a) as prepared, (b) $1 \mathrm{~h}$, (c) $2 \mathrm{~h}$, (d) $3 \mathrm{~h}$, (e) 1 day and (f) 4 days. The inset shows the fitted $\left(\begin{array}{lll}1 & 0 & 1\end{array}\right)$ reflection and the evolution of the FWHM with aging time.

Table 1. Particle size and band gap of synthesized Se nanorods.

Particle size (nm)

Aging period XRD analysis FESEM analysis Band gap $(\mathrm{eV})$

\begin{tabular}{lccc}
\hline As-prepared & - & - & $2 \cdot 24$ \\
$1 \mathrm{~h}$ & - & - & - \\
$2 \mathrm{~h}$ & $42 \cdot 16$ & - & - \\
$3 \mathrm{~h}$ & $40 \cdot 14$ & $41 \cdot 10$ & 2.26 \\
1 day & 39.60 & 39.70 & 2.72 \\
4 days & 41.88 & 42.18 & 2.41 \\
\hline
\end{tabular}

indicates high purity of the Se particles. The enhanced visibility of the XRD reflexes shown in figure 1(inset) represents that FWHM of the normalized prominent $\left(\begin{array}{lll}1 & 0 & 1\end{array}\right)$ peaks are changing as aging time proceeds. It indicates that the increase in crystallinity with $1 \mathrm{~h}, 3 \mathrm{~h}$ and 1 day aging with a shift is noticed at 1 day and absence of shift in 4 days aging. Lattice parameters are refined using the unit cell software and it is found to be $a=b=4.3614(5) \AA$ and $c=4$.9533(4) $\AA$.

The crystallite size of the Se particles is calculated using Scherrer's formula,

$$
D=0.94 \lambda / \beta \cos \theta,
$$


where $D$ is diameter of the particle, $\lambda$ the wavelength of incident radiation, $\beta$ the full width at half maximum (FWHM) and $\theta$ the diffraction angle. The calculated results are given in table 1. It is observed from the table that the average crystallite size decreases with increase in the aging time, but it increased at 4 days.

Figure 2( $\mathrm{a}$ and $\mathrm{b}$ ) shows the FTIR spectra of commercial Se $(99.9 \%)$ and synthesized Se nanoparticles, respectively. In figure 2(a), a medium intensity of band is shown at $465 \mathrm{~cm}^{-1}$ and a broad band is shown at $668 \mathrm{~cm}^{-1}$, which are due to the bending vibrations of $\mathrm{Se}-\mathrm{O}$ of $\mathrm{H}_{2} \mathrm{SeO}_{3}$. A sharp and strong intensity peak is shown at $1118 \mathrm{~cm}^{-1}$ due to stretching vibration of $\mathrm{SeO}_{2}$ (Chen et al 2006; Refat and Elsabawy 2011). Note that, these complexes may be formed during or after the synthesizing process. The FTIR spectrum of synthesized Se particles (figure $2 \mathrm{~b}$ ) is almost similar to that of commercial Se powder (figure 2a). However, the bending vibration of $\mathrm{Se}-\mathrm{O}$ band gets more broadened at $668 \mathrm{~cm}^{-1}$.

The effect of biomolecule on the surface morphology of $\mathrm{Se}$ particles is studied using FESEM images. It is observed from the micrograph (figure 3a) that a large number of Se rods are uniformly distributed over the surface. Average size of the rod is found to be $41 \mathrm{~nm}$ and length is several micrometres, which is clearly visible in the enlarged image of $3 \mathrm{~h}$ aging (figure $3 b$ ). In 1 day (figure 3c), the Se rods are attached with each other and seem like layered structure. It is entirely different surface morphology in comparison to the $3 \mathrm{~h}$ aging and it is developed more in the case of 4 days aging (figure $3 \mathrm{~d}$ ). The microstuctural changes are evident in the FESEM analysis due to the presence of $L$-arginine, which is periodically changing the shape and size of the Se in ethanol medium. On comparison with the early work, the Se nanowires were obtained using $\beta$-cyclodextrin at $2 \mathrm{~h}$ aging period ( $\mathrm{Li}$ and Yam 2006), whereas we have obtained the

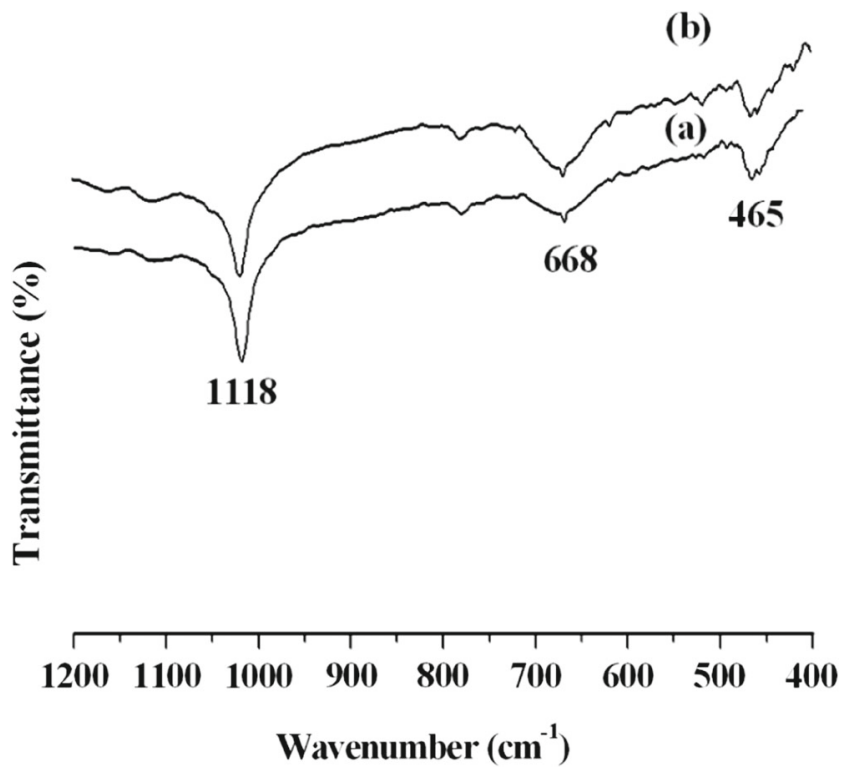

Figure 2. FTIR spectra of (a) commercial Se powder and (b) synthesized Se nanoparticles.
Se nanorods at $3 \mathrm{~h}$ aging. It is suggested that the formation of nanostructures mainly depends on the nature of the stabilizers. Hence, the $L$-arginine plays an important role not only in the formation of nanostructures, but also it is less harmful to the environment.

To study the optical absorption, certain amount of the synthesized Se particles was dissolved in hydrazine hydrate solution. From the recorded optical absorption spectra, the direct optical band gap was calculated using

$$
(\alpha h v)^{n}=A\left(h v-E_{\mathrm{g}}\right),
$$

where $E_{\mathrm{g}}$ is the energy gap, $\alpha$ the absorption coefficient, $h$ the Planck's constant, $v$ the frequency of light, $A$ the constant of proportionality and $n=2$ for direct band gap energy (Mehta et al 2008) and its results are shown in figure $4(\mathrm{a}-\mathrm{d})$. From the plots, band gap energy is found to be 2.24, 2.26, 2.72 and $2.41 \mathrm{eV}$ for as prepared, $3 \mathrm{~h}, 1$ day and 4 days aging times, respectively. It is observed that the band gap energy of synthesized Se nanostructures is higher than that of bulk $\alpha$-Se $(2.0 \mathrm{eV})$ and commercial Se powder $(1.8 \mathrm{eV})$ (Bhatnagar et al 1985) due to quantum size effect. Similar to the early report (Liao et al 2010), the band gap of t-Se was found to be blue shifted as the crystallite size is reduced from bulk to $41 \cdot 02,39 \cdot 70$, and $42 \cdot 18 \mathrm{~nm}$. As we have experienced for many other band gap semiconductors, quantum size effect of the Se nanorods should bring in new types of applications or enhance the performance of existing devices. The variations in band gap energy correspond to the size of Se nanorods as it can be correlated with the results of FESEM and XRD analyses.

In typical reaction mechanism (1)-(3), $L$-arginine is formed as cation, when it is dissolved in water, while $\mathrm{SeO}_{2}$ is formed as selenious acid $\left(\mathrm{H}_{2} \mathrm{SeO}_{3}\right)$ in the form of $\mathrm{H}^{+}$and $\mathrm{HSeO}_{3}^{-}$. Then, the two ions are combined to form arginine selenite (Kuvaeva et al 2007). Consequently, $L$-arginine protonate and $\mathrm{HSeO}_{3}^{-}$deprotonate in the solution are interacted with each other to form again $\mathrm{H}_{2} \mathrm{SeO}_{3}$. When ascorbic acid is added to the solution, it reduces $\mathrm{H}_{2} \mathrm{SeO}_{3}$ as $\mathrm{Se}$ ( $\mathrm{Li}$ and Yam 2006). In the absence of $L$-arginine in the solution, the ascorbic acid yields colloidal $\alpha$-Se due to its strong reducing property. The $\alpha$-Se species is gradually dissolved in absolute ethanol and starts crystallization as aging time proceeds.

$$
\begin{aligned}
& \mathrm{SeO}_{2}+\mathrm{H}_{2} \mathrm{O} \rightarrow \mathrm{H}_{2} \mathrm{SeO}_{3}, \\
& { }^{+} \mathrm{NH}_{3}-L \text {-arg }+\mathrm{HSeO}_{3}^{-} \rightarrow \mathrm{HSeO}_{3}^{-}+\mathrm{NH}_{3}-L \text {-arg } \\
& \rightarrow \mathrm{H}_{2} \mathrm{SeO}_{3}+\mathrm{NH}_{2}-L-\arg , \\
& \mathrm{H}_{2} \mathrm{SeO}_{3}+2 \mathrm{C}_{6} \mathrm{H}_{8} \mathrm{O}_{6} \rightarrow \mathrm{Se} \downarrow+2 \mathrm{C}_{6} \mathrm{H}_{6} \mathrm{O}_{6}+3 \mathrm{H}_{2} \mathrm{O},
\end{aligned}
$$

where

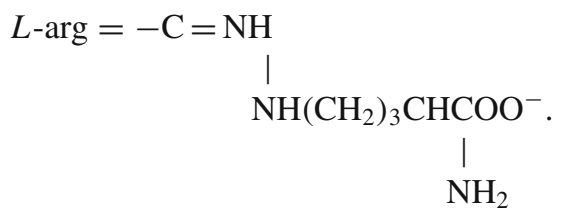




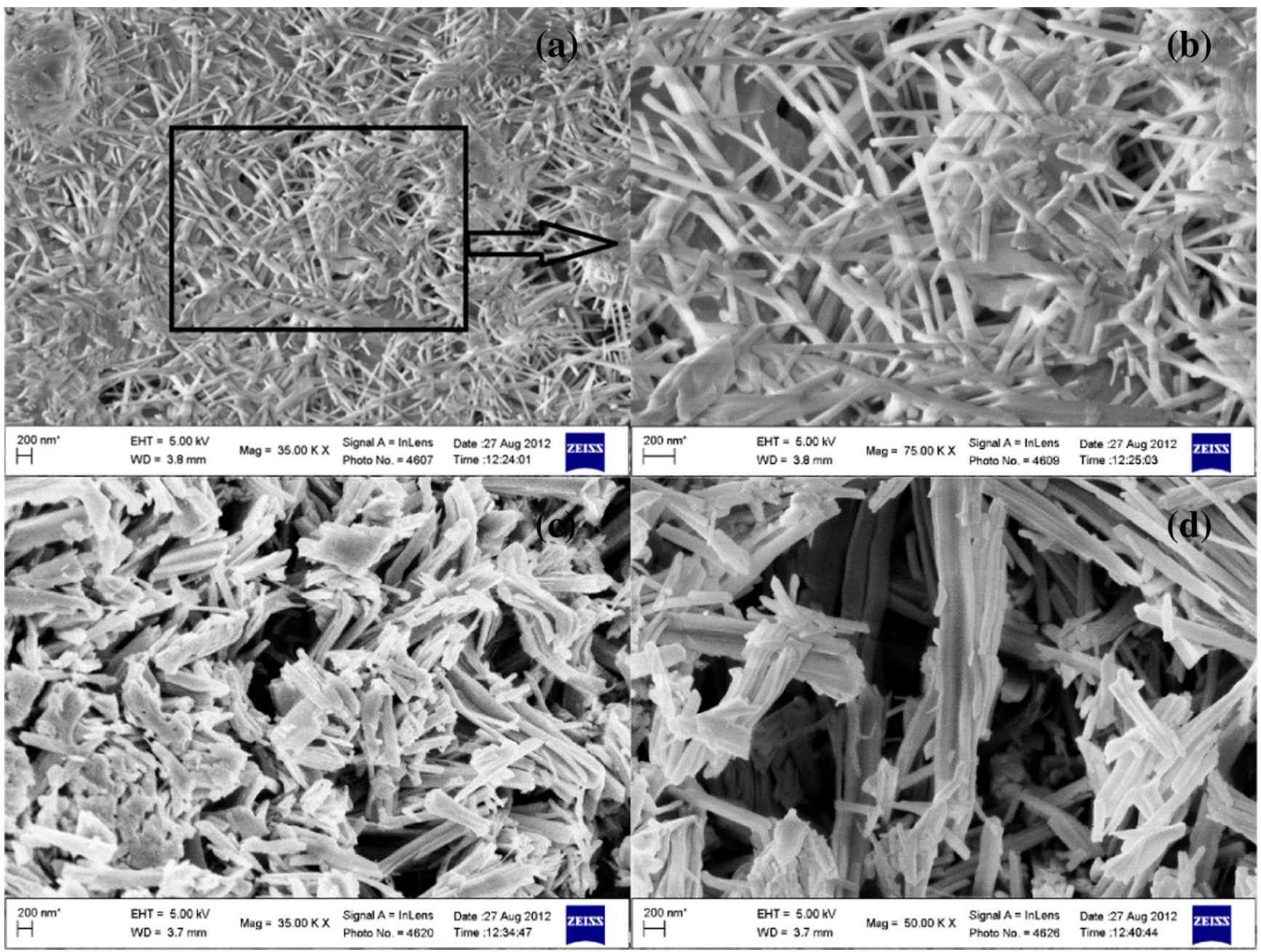

Figure 3. FESEM images of Se nanorods (a) 3 h, (b) enlarged image of $3 \mathrm{~h}$ (marked by rectangular box), (c) 1 day and (d) 4 days of aging.

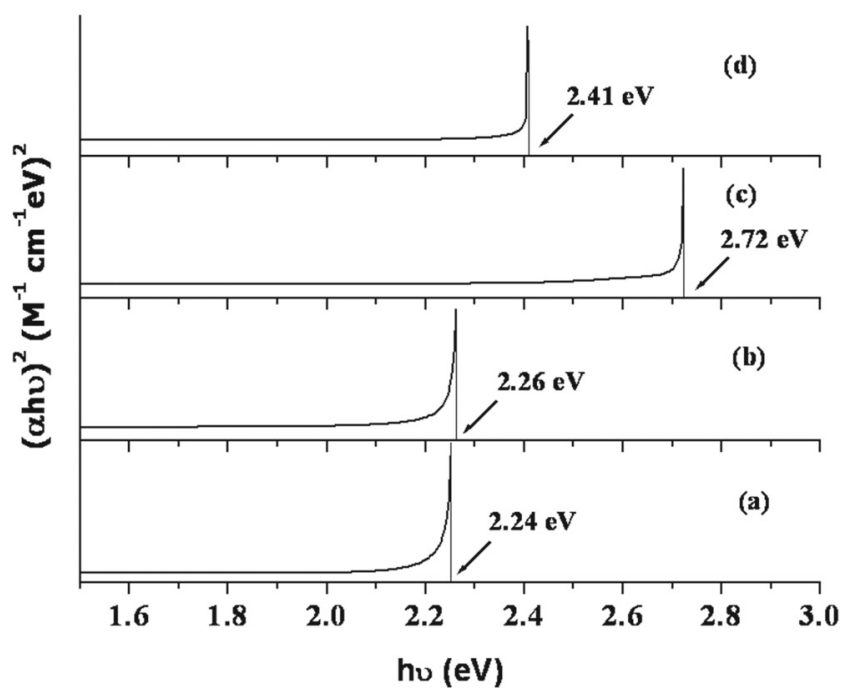

Figure 4. Plots between $(\alpha h v)^{2}$ and energy $(h v)$ for synthesized Se nanoparticles: (a) as prepared, (b) 3 h, (c) 1 day and (d) 4 days of aging.

\section{Conclusions}

Nanorods of 1D trigonal selenium (t-Se) are synthesized using $L$-arginine and ascorbic acid for different aging times by precipitation method. XRD pattern indicates that the $\alpha$-Se transformed into t-Se at $3 \mathrm{~h}$ ethanol medium. Crystallinity is found to be increased up to 1 day aging time and it decreased at 4 days aging time. Average crystallite size of the Se nanorods is found to be $42 \cdot 16,40 \cdot 14,39 \cdot 82$ and $41.88 \mathrm{~nm}$ for $2 \mathrm{~h}, 3 \mathrm{~h}, 1$ day and 4 days aging times, respectively. The FTIR spectra confirmed the presence of stretching and bending vibrations of $\mathrm{Se}-\mathrm{O}$ in both Se samples at 465,668 and $1118 \mathrm{~cm}^{-1}$. The FESEM photographs are evidenced for the changes of Se rod size in the ethanol medium. Average size of Se nanorods is found to be 41.02 , 39.70 and $42.18 \mathrm{~nm}$ for $3 \mathrm{~h}, 1$ day and 4 days aging times, respectively. Optical band gap energy of $\alpha$-Se and t-Se nanoparticles are found to be $2 \cdot 24,2.26,2.72$ and $2.41 \mathrm{eV}$ for as-prepared, 3 h, 1 day and 4 days aging times, respectively. The calculated band gap energy is larger than the bulk $\alpha$-Se and crystalline Se. In this study, pure 1D Se nanorods are obtained using the biomolecule substances which are 
less harmful due to their biological nature and required no heat treatment and also low-cost production. However, further investigation is needed to determine the changes of Se nanostructures beyond 4 days of aging time.

\section{Acknowledgements}

The authors express their sincere thanks to the UGC-SAP, New Delhi and authorities of Manonmaniam Sundaranar University, Tirunelveli, for providing the seed money project to carry out the research work.

\section{References}

Abdelouas A, Gong W L, Lutze W, Shelnutt J A, Franco R and Moura I 2000 Chem. Mater. 121510

Bhatnagar A K, Reddy K V and Srivastava V 1985 J. Phys. D: Appl. Phys. 18 L149

Brown R D 2000 US Geological Survey, Minerals Year Book 67.1

Chen H, Shin D, Nam J, Kwon K and Yoo J 2010 Mater. Res. Bull. 45699

Chen X, Li H, Wang S, Yang M and Qi Y 2012 Mater. Lett. 6622

Chen Y, Li L, D’Ulivo A and Belzile N 2006 Analy. Chim. Acta 577126

Choi K and Lee J 2011 Sci. Adv. Mater. 3811

Gao X, Gao T and Zhang L 2003 J. Mater. Chem. 136

Gao F, Lu Q, Meng X and Komarneni S 2008 J. Mater. Sci. 432377

Huang T and Qi L 2009 Nanotechnology 2025606
Kislyuk V V and Dimitriev O P 2008 J. Nanosci. Nanotechnol. 8 131

Kuvaeva Z I, Lopatik D V, Popova O P, Pokrovskaya A I, Lysenko G N and Markovich M M 2007 Pharmaceut. Chem. J. 41157

Li Q and Yam V W 2006 Chem. Commun. 1006

Liao Z M, Hou C, Liu L P and Yu D P 2010 Nanoscale Res. Lett. 5926

Liu L, Peng Q and Li Y 2008 Nano Res. 1403

Ma Y, Qi L, Ma J and Cheng H 2004 Adv. Mater. 161023

Mehta S K, Chaudhary S, Kumar S, Bhasin K K, Torigoe K, Sakai $\mathrm{H}$ and Abe M 2008 Nanotechnology 19295601

Refat M S and Elsabawy K M 2011 Bull. Mater. Sci. 34873

Shah C P, Kumar M and Bajaj P N 2007 Nanotechnology 18 385607

Song J, Zhu J and Yu S 2006 J. Phys. Chem. B 11023790

Tong H, Zhu Y, Yang L, Li L and Zhang L 2006 Angew. Chem. Int. Ed. 457739

Wang X, Zhang W, Shen Y, Xie A and Huang L 2011 Mater. Sci. Eng. B 1761093

Zhang J, Zhang S Y, Xu J J and Chen H Y 2004 Chin. Chem. Lett. 151345

Zhang B, Ye X, Dai W, Hou W, Zuo F and Xie Y 2006a Nanotechnology 17385

Zhang B, Ye X, Hou W, Zhao Y and Xie Y 2006b J. Phys. Chem. B 1108978

Zhang X Y, Xu L H, Dai J Y, Cai Y and Wang N 2006c Mater. Res. Bull. 411729

Zhu W, Xu H, Wang W and Shi J 2006 Appl. Phys. A 83281

Zingaro R A and Copper W C 1974 Selenium (New York: Van Nostrand-Reinhold) 